\title{
ADAPTACIÓN DEL FRIJOL A SUELOS DE BAJA FERTILIDAD BAJO PRESIÓN DE ENFERMEDADES FOLIARES EN COSTA RICA ${ }^{1}$
}

\author{
Rodolfo Araya ${ }^{2}$, Jorge A. Acosta ${ }^{3}$
}

\begin{abstract}
RESUMEN
Adaptación del frijol a suelos de baja fertilidad bajo presión de enfermedades foliares en Costa Rica. El objetivo de este estudio fue identificar germoplasma y líneas mejoradas de alta productividad, en suelos de baja fertilidad y bajo presión de antracnosis y mancha angular. Durante 1996 y 1997 se evaluaron fuentes de tolerancia a la baja fertilidad de diversa procedencia geográfica y con resistencia para otros factores bióticos y abióticos. Se caracterizaron 562 genotipos, 251 mejorados y 311 criollos, en dos localidades de Costa Rica: Fraijanes (1750 msnm; 15,3 ${ }^{\circ} \mathrm{C} ; 3025 \mathrm{~mm} ; 10^{\circ} 09^{\prime}$ Latitud norte y $84^{\circ} 12^{\prime}$ Longitud oeste) y Alajuela ( $840 \mathrm{msnm}, 23,1$ ${ }^{\circ} \mathrm{C} ; 1966 \mathrm{~mm} ; 10^{\circ} 01^{\prime}$ Latitud norte y 84 $16^{\prime}$ ' Longitud oeste). La densidad de siembra fue de 15 semillas por metro lineal y el tamaño de parcela varió con base en la disponibilidad de semilla; a la siembra sólo se adicionó inoculante de Rhizobium tropici al surco de siembra (4 kg/ha). La línea A321, el testigo local UCR 55, A483, G11640; G19696, G10843 y BAC50 mostraron alto rendimiento en las dos localidades. Los genotipos de alto rendimiento en una localidad fueron: en Alajuela: Guanajuato 132, Negro INIFAP, VAX 2, MAR 3 y en Fraijanes G13860, G92, MOC112, Bayo Alteño, J 117; Negro 8025 y A 247. Se identificó germoplasma no mejorado de diferente acervo genético, procedente de México, Perú y Guatemala, con tolerancia a suelos de baja fertilidad. Esto indica la importancia de evaluar material criollo y del intercambio de germoplasma entre países. Los genotipos superiores serán utilizados en el mejoramiento del frijol común para agricultura de bajo uso de insumos.
\end{abstract}

\begin{abstract}
Adaptation of bean to low fertility soils under foliar diseases pressure in Costa Rica. The aim of this study was to identify bean genotypes with high yield potential when grown in soils of low fertility and under the pressure of foliar diseases. Diverse genotypes, known for its adaptation in soils of low fertility and/or resistance to other biotic and abiotic stresses were evaluated in the field during 1996 and 1997. In total, 562 genotypes, 251 bred and 311 landraces, were evaluated in two locations in Costa Rica: Fraijanes (1750 $\mathrm{msnm} ; 15.3^{\circ} \mathrm{C}$; $3025 \mathrm{~mm}$ annual precipitation; $10^{\circ} 09^{\prime}$ North and $84^{\circ} 12^{\prime}$ 'West); and Alajuela (840 msnm, $23,1^{\circ} \mathrm{C}$; 1966 $\mathrm{mm}$ annual precipitation; $10^{\circ} 01^{\prime}$ North and $84^{\circ} 16^{\prime}$ 'West). Plant stand was of 15 seeds per lineal $\mathrm{m}$ and plot size was variable due to seed availability; at planting inoculant of Rhizobium tropici was added directly onto the seed in the row (4 kg/ha). Bred line A 321, the control genotype UCR 55, A 483, G11640, G19696, G10843 and BAC50 displayed high yields in both locations. Genotypes that showed high yield in only one location were, in Alajuela: Guanajuato 132, Negro INIFAP, VAX 2 and MAR 3; in Fraijanes higher yielders were G13860, G92, MOC 112, Bayo Alteño, J 117; Negro 8025 and A 247. Outstanding landraces and bred lines from diverse geographical areas and genetic pools that displayed adaptation in soils of low fertility were identified in germplasm introduced from CIAT, México, Perú and Guatemala. It is emphasized the importance of evaluating native material and the exchange of germplasm among countries. Superior genotypes will be used for bean breeding in low input agriculture.
\end{abstract}

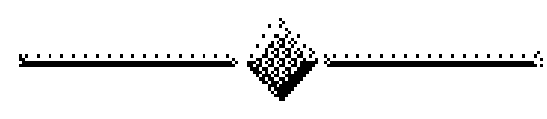

\section{INTRODUCCIÓN}

En Costa Rica predominan los suelos de baja fertilidad (Corella, 1983) con bajo contenido de fósforo, igual o menor a 10 ug de fósforo/ml (Bertsch, 1987).
Por ello, es necesario identificar genotipos de frijol con adaptación a esas condiciones edáficas. Las áreas en donde se siembra frijol están dominadas por pequeños agricultores, los que emplean reducidas cantidades de agroquímicos e inclusive no emplean fertilizantes, co-

\footnotetext{
1. Investigación financiada por la Universidad de Costa Rica, Vicerrectoría de Investigación y El Programa Cooperativo Regional de Frijol para Centroamérica, México y El Caribe (PROFRIJOL).

2. Universidad de Costa Rica, Estación Experimental Fabio Baudrit Moreno. Apartado 183-4050 Alajuela, Costa Rica. E-mail: avillalo@cariari.ucr.ac.cr

3. Programa de Frijol del INIFAP, Apartado Postal 10, Texcoco, México, CP56230, México.
} 
mo es el caso del frijol tapado (González y Araya, 1994). En estudios previos no fue posible obtener genotipos de frijol, con un nivel superior de tolerancia genética a suelos de baja fertilidad a través de cruzamientos (Singh et al. 1989), lo que sugiere ampliar la base genética si se pretende mejorar la adaptación a suelos pobres. Por otro lado, investigaciones sobre identificación de genotipos de frijol tolerantes a una moderada disponibilidad de fósforo con base a la producción de biomasa de la parte aérea y semilla indicaron la existencia de una amplia variación entre genotipos de diferentes orígenes (Acosta et al.), 1995).

En el trópico bajo de México se encontró evidencia de que genotipos del altiplano, mostraron adaptación a suelos ácidos con baja disponibilidad de fósforo (López et al. 1995). Las diferencias entre genotipos de frijol en capacidad de extracción de fósforo del suelo o por su alta eficiencia en la utilización de este elemento, indican que esa variabilidad genética puede ser utilizada en el mejoramiento genético de la especie (Posada et al. 1995; Muñoz y Beck, 1995; Lynch et al. 1995; Corella 1995; Ribet et al. 1997).

La fertilización química es sólo una solución parcial en los suelos de baja fertilidad, debido a la fijación de fósforo en las arcillas del suelo y por la lixiviación del nitrógeno en áreas de alta precipitación. En el desarrollo de variedades de frijol para una región específica se sugiere, dentro de la estrategia de mejoramiento, tomar en cuenta, además de los factores bióticos, los diversos factores abióticos que ocurren en la misma, debido a que varios de ellos están interrelacionados, como son la disponibilidad del fósforo, la fijación de nitrógeno y la sequía. La tolerancia a todos estos factores en parte depende de un buen sistema radical.

La obtención de variedades para uso comercial implica la evaluación de germoplasma bajo las condiciones de baja fertilidad promedio de la zona frijolera. En cada región agro ecológica, las fuentes de tolerancia a suelos pobres se tienen que valorar para determinar su adaptación y estabilidad. En la actualidad la diferencia entre un genotipo tolerante y uno susceptible es pequeña y la ventaja se pierde fácilmente por la mala adaptación a un sitio de prueba (Ribet et al. 1997). La estabilidad de los genotipos debe ser prioritaria, ya que hay mucha variación entre localidades y años. La precocidad fenológica, es una característica que es parte de la capacidad de un genotipo para escapar a cualquier estrés que se incremente al final del ciclo del cultivo, sin embargo, ésta característica no es de utilidad en suelos de baja fertilidad.

La selección de nuevas fuentes de tolerancia a baja fertilidad y a enfermedades es básica, para lograr el objetivo de brindar a los pequeños productores de frijol una variedad con tolerancia a factores bióticos y de mayor productividad en condiciones de bajos insumos y suelos pobres. El objetivo de esta investigación fue identificar genotipos de frijol de alto potencial de rendimiento, en suelos de baja fertilidad y bajo incidencia de las enfermedades fungosas: antracnosis y mancha angular. La línea mejorada UCR 55, con adaptación a condiciones de bajo fósforo y resistente a las enfermedades mencionadas, se utilizó como testigo para comparación.

\section{MATERIALES Y MÉTODOS}

\section{Localidades de prueba}

El germoplasma estudiado se sembró en dos localidades de Costa Rica, Fraijanes (Sub-Estación Experimental Fraijanes de la Universidad de Costa Rica, ubicada a $10^{\circ} 09^{\prime}$ latitud norte y $84^{\circ} 12^{\prime}$ ' longitud oeste a $1850 \mathrm{msnm}$ ) y Alajuela (Estación Experimental Fabio Baudrit Moreno, ubicada a $10^{\circ} 01^{\prime}$ de latitud norte y $84^{\circ} 16^{\prime}$ de longitud oeste a $840 \mathrm{msnm}$ ). Se definieron dos épocas de siembra: mayo y setiembre en las dos localidades en los años de 1996 y 1997. Los suelos donde se ubicaron los experimentos en la Estación Experimental Fabio Baudrit, pertenecen a la clase Ferric Luviasol (Clasificación FAO-UNESCO, 1973) y se clasifican como inceptisoles de textura areno arcilloso (Lynch y Sawyer, 1989) (Cuadro 1). En Fraijanes los suelos poseen como material matriz cenizas volcánicas recientes, clasificado como Hydric Dystrandept tixotrópico isotérmico, de baja fertilidad y profundo (Vásquez, 1982). El suelo del sitio experimental en particular y de la región en general, posee un $\mathrm{pH}$ bajo menor a 5,5 y la disponibilidad de fósforo en su mayoría es menor a $10 \mathrm{ug} / \mathrm{ml}$, considerado bajo (Bertsch, 1987).

Cuadro 1. Características de los suelos en dos localidades de Costa Rica.

\begin{tabular}{l|c|cccc|ccccc}
\hline Identificación* & $\mathbf{p H}$ & \multicolumn{4}{|c|}{ Meg/100 } & $\mathbf{m l}$ suelo & \multicolumn{5}{c}{ Ug/ml suelo } \\
& & AL & $\mathbf{C a}$ & $\mathbf{M g}$ & $\mathbf{K}$ & $\mathbf{P}$ & $\mathbf{Z n}$ & $\mathbf{M n}$ & $\mathbf{C u}$ & $\mathbf{F e}$ \\
\hline \multirow{2}{*}{ Fraijanes } & 5,0 & 0,3 & 4,0 & 1,0 & 0,40 & 10 & 5,8 & 5 & 5 & 93 \\
Alajuela & 5,3 & 0,7 & 3,8 & 1,0 & 0,26 & 12,6 & 1,5 & 23,1 & 12,3 & 136
\end{tabular}

*/ Fraijanes suelos derivados de cenizas volcánicas. Alajuela, suelos aluviales.

Germoplasma utilizado

Durante 1996 y 1997 se evaluaron 562 materiales: 251 mejorados y 311 criollos. Estos últimos proceden- 
tes de una selección previa en suelo de baja fertilidad efectuada en Fraijanes, Costa Rica. El germoplasma mejorado pertenecía a diferentes viveros: 145 materiales de un Vivero de Fuentes de Resistencia (CIAT 1996); 26 líneas de un Vivero de Bajo Fósforo de México (INIFAP); 60 líneas de frijol con adaptación a suelos ácidos y de baja fertilidad (líneas provenientes de México y Costa Rica); 83 materiales del Vivero de Líneas Promisorias de Frijol para bajo Fósforo (vivero compuesto por material seleccionado en México, Costa Rica y CIAT) (Saborío y Beebe, 1995); 57 genotipos de la colección representativa de la variabilidad de $P$. vulgaris (CORE Collection) seleccionados para bajo fósforo (CIAT 1995); 162 accesiones de germoplasma nativo de Costa Rica, y 29 genotipos del Vivero Internacional de Fuentes de Resistencia a la Antracnosis (CIAT 1996). Como testigo para comparación de rendimiento se utilizó en ambas localidades la línea UCR 55, línea sobresaliente entre los materiales evaluados en Costa Rica, ya que es resistente a la antracnosis, tolerante a la mancha angular y de alta producción en suelos de baja fertilidad. Otros genotipos utilizados como testigos fueron BAT 76, DOR 390 y ANT 7, que han mostrado buen rendimiento en condiciones de baja fertilidad. El tamaño de la parcela fue variable dependiendo de la disponibilidad de semilla, de tres a cuatro surcos de cuatro a seis metros de largo, sin repeticiones. La distancia entre surcos fue de $60 \mathrm{~cm}$ y se sembraron 15 semillas por metro lineal. Cada diez surcos de material experimental se establecieron los testigos. No se empleó fertilizante y sólo se adicionó inoculante de Rhizobium tropici sobre la semilla al momento de la siembra.

\section{Características cuantificadas}

Se determinaron las características fenológicas del germoplasma y su reacción a las enfermedades y como indicador de tolerancia a la baja fertilidad del suelo, se comparó el rendimiento de cada genotipo con el rendimiento del testigo UCR 55 (100\%) más cercano. Otro factor de selección se basó al considerar el efecto reductor del rendimiento por las enfermedades, antracnosis y mancha angular; así, los genotipos con rendimiento superior o similar al de la línea UCR 55, indicaría no sólo tolerancia a los dos patógenos sino también tolerancia a la baja fertilidad.

\section{RESULTADOS}

En ambas localidades y siembras, hubo condiciones climáticas (Cuadro 2) que favorecieron el desarrolo de las enfermedades inoculadas y en todas las evaluaciones, la mayoría de los genotipos estudiados resultaron susceptibles a una o a las dos enfermedades. Es probable que además de las razas inoculadas, hayan ocurrido otras razas en forma natural, lo cual provocó una fuerte presión por esas enfermedades. No hubo genotipos resistentes a la mancha angular y sólo unos cuantos resultaron resistentes a la antracnosis.

En los dos sitios de evaluación, los materiales consistentes en rendimiento fueron: G 11640; G 19696, ambos de hábito trepador, de gran desarrollo vegetativo en baja fertilidad en Alajuela y respuesta intermedia en Fraijanes; G 10843, BAC 50 (de grano rojo brillante y precoz) y el testigo UCR 55 (Cuadro 3). En Fraijanes se observaron los más altos rendimientos y entre los materiales más sobresalientes, A 321 y el testigo UCR 55, mostraron alto rendimiento en los dos sitios de prueba, sitios contrastantes por su clima y condiciones edáficas. A 321 resultó, sin embargo, susceptible a las enfermedades. A 321 ha sido también identificado como tolerante a suelos de bajo contenido de fósforo en África (Wortmann et al. 2000). Otros genotipos que mostraron un rendimiento promedio de intermedio a alto fueron: A 483; VAX 2, MAR 3, J 117, MAR 3, Manzano, variedad mejorada de la región semiárida de México de

Cuadro 2. Condiciones climáticas imperantes durante el periodo en que los ensayos estuvieron sembrados a nivel de campo, en las localidades de Fraijanes y Alajuela.

\begin{tabular}{|c|c|c|c|c|c|c|c|c|c|c|c|c|c|c|c|c|}
\hline \multicolumn{17}{|c|}{ Fraijanes * } \\
\hline & mayo & junio & julio & $\begin{array}{l}1996 \\
\text { agosto }\end{array}$ & set & oct & nov & dic & mayo & junio & julio & $\begin{array}{c}1997 \\
\text { agosto }\end{array}$ & set & oct & nov & dic \\
\hline Temperatura $\left({ }^{\circ} \mathrm{C}\right)$ & 18 & 18 & 17 & 17 & 17 & 16 & 16 & 16 & 17 & 17 & 17 & 18 & 17 & 18 & 17 & 17 \\
\hline Humedad (\%) & 91 & 90 & 89 & 87 & 89 & 92 & 90 & 85 & 89 & 89 & 88 & 86 & 88 & 90 & & 84 \\
\hline Lluvia (mm) & 449 & 319 & 370 & 575 & 680 & 375 & 388 & 176 & 307 & 464 & 215 & 349 & 595 & 333 & 358 & 162 \\
\hline \multicolumn{17}{|c|}{ Alajuela * } \\
\hline Temperatura (\%) & 23 & 23 & 21 & 23 & 23 & 22 & 23 & 23 & 24 & 23 & 25 & 25 & 23 & 24 & 23 & 24 \\
\hline Humedad (\%) & 85 & 87 & 85 & 82 & 89 & 89 & 82 & 68 & 74 & 84 & 74 & 73 & 85 & 84 & 86 & 69 \\
\hline Lluvia (mm) & 337 & 225 & 353 & 250 & 334 & 222 & 195 & 5 & 187 & 302 & 71 & 121 & 424 & 337 & 382 & 2 \\
\hline
\end{tabular}

_/ Programa de Agroambiente. Estación Experimental Fabio Baudrit Moreno. Universidad de Costa Rica.

*/ Temperatura y humedad promedio, y lluvia acumulada por mes. 
Cuadro 3. Caracteres agronómicos y rendimiento (porcentaje en relación con testigo UCR 55) de genotipos de frijol sobresalientes en suelos de baja fertilidad evaluados en dos localidades de Costa Rica, en 1996 y 1997.

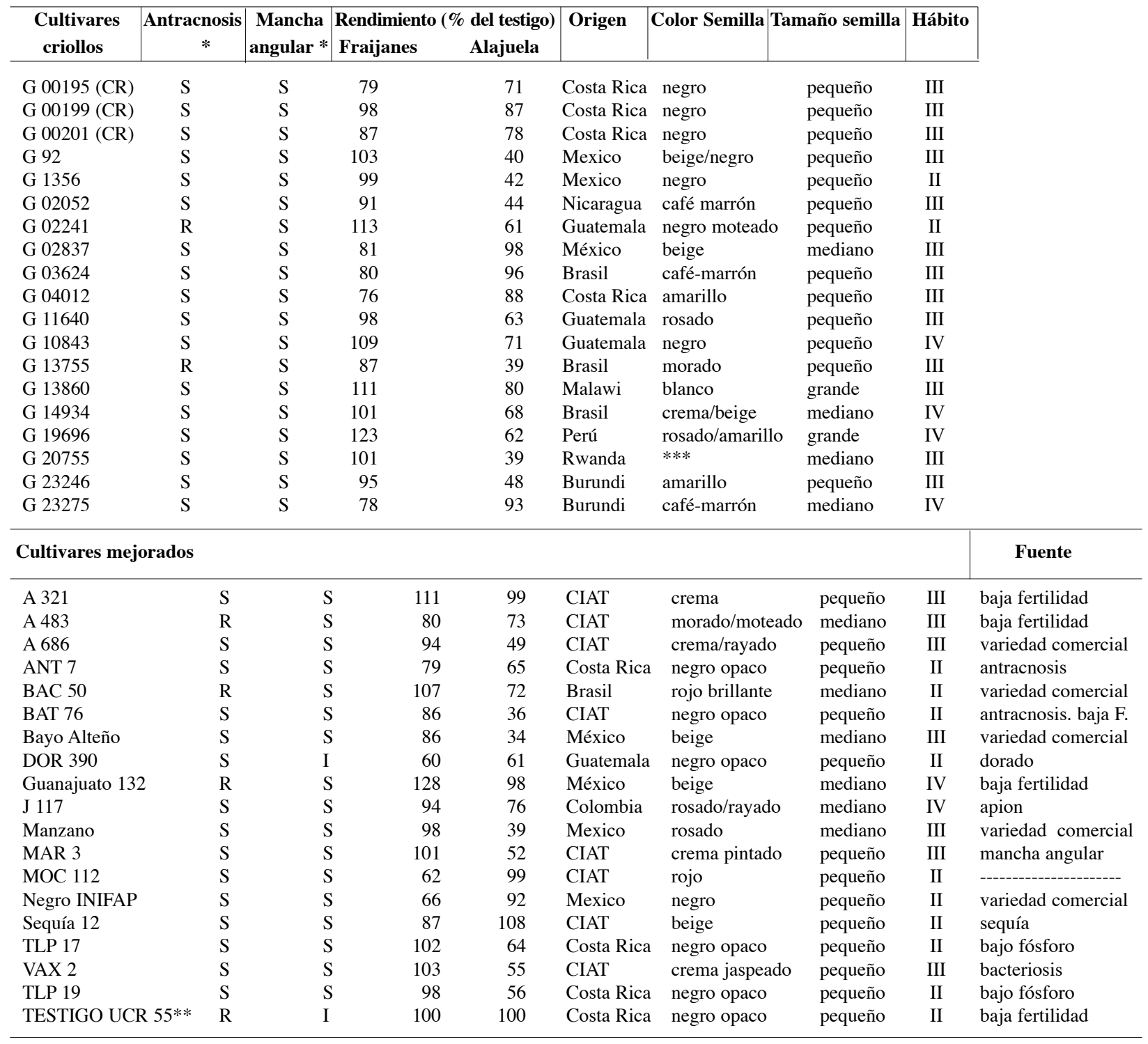

$* / . \mathrm{R}=$ resistente, $\mathrm{S}=$ susceptible

**/. Rendimiento de UCR 55 = Fraijanes 570 a $890 \mathrm{~kg} / \mathrm{ha}$. Alajuela 711 a $1110 \mathrm{~kg} / \mathrm{ha}$.

*** tipo variedad fustula de chile.

hábito indeterminado tipo III; G 13860, G 92; MOC 112 (de grano rojo precoz); Bayo Alteño, variedad mejorada del Altiplano sub-húmedo de México, de hábito indeterminado tipo III. La variedad comercial Catrachita (variedad comercial precoz de grano de color rojo desarrollada en Honduras), que se está utilizando como progenitor en los programas de mejoramiento en Centroamérica, manifestó una respuesta intermedia en suelos de baja fertilidad.

El genotipo Guanajuato 132 (semilla de color crema y de ciclo precoz) se destacó por su alta productividad en la localidad de Alajuela. En esta localidad los genotipos con un rendimiento intermedio fueron los si- guientes: G 14934 (de ciclo biológico tardío); G 1356; Negro INIFAP (de grano negro opaco y excelente desarrollo vegetativo), G 23246 y G 20755. Los genotipos de grano rojo son de especial interés ya que ésta es una de las clases comerciales preferidas en el país.

\section{DISCUSION}

La identificación de germoplasma con adaptación a baja fertilidad, procedente de diversos países como México, Perú y Guatemala (Cuadro 3), indican la existencia de tolerancia a suelos de baja fertilidad en diferentes acervos genéticos y la importancia de evaluar 
material criollo. Lo anterior evidencia el proceso de mejoramiento realizado para bajos insumos por los agricultores a través de generaciones. Los genotipos mejorados para baja fertilidad en otras latitudes mantuvieron su adaptación en suelos de baja fertilidad y materiales mejorados para factores bióticos, mostraron como nueva característica su tolerancia a la baja fertilidad. Sin embargo, y debido a la alta presión ejercida por las enfermedades, la mayoría de los genotipos en ambos grupos resultaron susceptibles a las enfermedades. El siguiente paso será el realizar cruzamientos entre los mejores genotipos en ambas características, adaptación a suelos de baja fertilidad y resistencia a enfermedades.

En la presente investigación, se encontró que todos los genotipos con adaptación a suelos de baja fertilidad en las dos localidades de prueba, fueron de hábito de crecimiento indeterminado, de los tipos II, III y IV. Resultados similares fueron encontrados por Wortmann et al. (2000), quienes indicaron que los genotipos de hábito determinado pueden mostrar adaptación a suelos ácidos, pero no a suelos pobres en nitrógeno y fósforo. $\mathrm{Si}$ el crecimiento de la parte aérea refleja el crecimiento del sistema radical, entonces los genotipos de hábito indeterminado poseen un sistema radical más ramificado, el cual permite una mejor adaptación a los suelos pobres en comparación con los genotipos de hábito determinado.

La investigación sobre factores abióticos, no debe efectuarse con el propósito de lograr la obtención de variedades comerciales resistentes a un sólo factor en particular para una región determinada, debido a la incidencia de otros factores, tanto abióticos o bióticos, para los cuales el germoplasma presenta susceptibilidad. Con base en este criterio el mejoramiento para factores abióticos, se debería realizar en etapas avanzadas, por ejemplo, después de identificar genotipos resistentes a las enfermedades. Las líneas y genotipos introducidos con resistencias incorporadas, podrían utilizarse en el mejoramiento para caracteres específicos a un país o zona, donde este factor abiótico sea importante.

\section{CONCLUSIONES}

Con base en el rendimiento, se identificaron genotipos de frijol de diferente origen geográfico y acervo genético con adaptación a los suelos pobres de Fraijanes y Alajuela, Costa Rica.

La mayoría de los genotipos evaluados resultaron susceptible a la antracnosis y a la mancha angular, enfermedades fungosas de alta incidencia en las localidades de prueba.

\section{LITERATURA CITADA}

ACOSTA-GALLEGOS, J. A.; ACOSTA-DÍAZ, ALVARADO, E.; S. 1995. Identificación de genotipos de frijol con adaptación a suelos de baja fertilidad. In: Mejoramiento y manejo agronómico del frijol común (Phaseolus vulgaris L.) para adaptación en suelos de bajo fósforo: memoria del Taller Internacional sobre Bajo Fósforo en el cultivo del frijol. San José, C.R. Universidad de Costa Rica. pp. 26-34.

BECK, D.; MUÑOZ, F. 1997. Manejo de la variabilidad espacial y niveles de stress en suelos para evaluación de tolerancia del frijol a bajo fósforo. In: Mejoramiento y manejo agronómico del frijol común (Phaseolus vulgaris L.) para adaptación en suelos de bajo fósforo: memoria del Taller Internacional sobre Bajo Fósforo en el cultivo del frijol. San José, C.R. Universidad de Costa Rica. pp. 139149.

BEEBE, S. 1995. Mejoramiento genético del frijol (Phaseolus vulgaris L.) para tolerancia a bajo fósforo: estrategias y fuentes de diversidad. In: Mejoramiento y manejo agronómico del frijol común (Phaseolus vulgaris L.) para adaptación en suelos de bajo fósforo: memoria del Taller Internacional sobre Bajo Fósforo en el cultivo del frijo. San José, C.R. Universidad de Costa Rica. pp.1-8.

BERTSCH, F. 1987. Manual para interpretar la fertilidad de los suelos de Costa Rica. San José, C.R. Universidad de Costa Rica. 78 p.

CORELLA, J. 1995. Recomendaciones de manejo de la fertilización del cultivo de frijol en Costa Rica. In: Mejoramiento y manejo agronómico del frijol común (Phaseolus vulgaris L.) para adaptación en suelos de bajo fósforo: memoria del Taller Internacional sobre Bajo Fósforo en el cultivo del frijol, 13-15 noviembre de 1995. San José, C.R. Universidad de Costa Rica. pp.110-138.

FAO. 1973. Costa Rica. Organización de distritos de riego en la cuenca del Itiquis: suelos. Informe preliminar. Conclusiones y recomendaciones. Roma, pp. 29-29.

GONZALEZ, W.; ARAYA, R. 1994. Estudio agroeconómico del frijol (Phaseolus vulgaris L.) Tapado en Costa Rica. In: Tapado: los sistemas de siembra con cobertura. Ed. H. David Thurston, Margaret Smith, George Abawi y Steve Kearl. Ithaca, New York. Cornell International Institute for Food, Agriculture and development (CIIFAD) y CATIE. $329 \mathrm{p}$.

LÓPEZ-SALINAS, E; CUMPÍAN, J.; DURÁN, A.; ACOSTAGALLEGOS, J.A. 1995. Tolerancia del frijol común a la baja disponibilidad de fósforo en suelos de Veracruz, México. In: Mejoramiento y manejo agronómico del frijol común (.Phaseolus vulgaris L.) para adaptación en suelos de bajo fósforo: memoria del Taller Internacional sobre Bajo Fósforo en el cultivo del frijol. San José, C.R. Universidad de Costa Rica. pp. 35-40. 
LYNCH B.; SAWYER, S. 1989. Classification and distribution of soils of the Fabio Baudrit Experimental Station. Edt. Larry Dyer. San José, Costa Rica. Pirr.

LYNCH J.; NIELSEN, K.L.; EBEL, R. ; BONSER, A.; BECK, D. 1995. La adaptación fisiológica de frijol común (Phaseolus vulgaris L.) a baja disponibilidad de fósforo. In Mejoramiento y manejo agronómico del frijol común (Phaseolus vulgaris L.) para adaptación en suelos de bajo fósforo: memoria del Taller Internacional sobre Bajo Fósforo en el cultivo del frijol. San José, C.R. Universidad de Costa Rica. pp. 58-75.

MUÑOZ, F.; BECK, D. 1995. Investigaciones efectuadas en CIAT sobre fuentes de mecanismos de tolerancia a bajo fósforo en frijol común: 1995. In: Mejoramiento y manejo agronómico del frijol común (Phaseolus vulgaris L.) para adaptación en suelos de bajo fósforo: memoria del Taller Internacional sobre Bajo Fósforo en el cultivo del frijol. San José, C.R. Universidad de Costa Rica. pp. 85-94.

POSADA, H; KORNEGAY, J.; BECK, D. 1995. Estudios de herencia y mecanismos de tolerancia en líneas de frijol (Phaseolus vulgaris L.) andinas y mesoamericanas a bajo fósforo en el suelo. In: Mejoramiento y manejo agronómico del frijol común (Phaseolus vulgaris L.) para adaptación en suelos de bajo fósforo: memoria del Taller
Internacional sobre Bajo Fósforo en el cultivo del frijol. San José, C.R. Universidad de Costa Rica. pp. 41-57.

ROSAS .J C.; ANREWS, M.; CASTRO, A.. 1995. Tolerancia del frijol común a la baja disponibilidad de fósforo en suelos de Honduras. In: Mejoramiento y manejo agronómico del frijol común (Phaseolus vulgaris L.) para adaptación en suelos de bajo fósforo: memoria del Taller Internacional sobre Bajo Fósforo en el cultivo del frijol. San José, C.R. Universidad de Costa Rica. pp.19-25.

SABORÍO, A.; BEEBE, S. 1995. Mejoramiento para tolerancia a bajo fósforo en Costa Rica. In: Mejoramiento y manejo agronómico del frijol común (Phaseolus vulgaris L.) para adaptación en suelos de bajo fósforo: memoria del Taller Internacional sobre Bajo Fósforo en el cultivo del frijol. San José, C.R. Universidad de Costa Rica. pp. 9-18.

VÁZQUEZ, M. A. 1982. Estudio detallado de suelos: Sub estación experimental de cultivos de altura Fraijanes. San José, Costa Rica. Vicerrectoría de Investigación, Universidad de Costa Rica. 32 p.

WORTMANN, C.S.; LUNZE, L.; CHIRWA, R.; RACHIER, G.; RABARY, B.; NDAKIDEMI, P.; MKUCHU, M.; MUIMUI, M.; NABUHUNGO, L. 2000. Bean improvement for low fertility soils in Africa: THE BILFA II. Ann. Rep. Bean Improv. Coop. 43:11-12 